Research Paper

\title{
High expression of calreticulin indicates poor prognosis and modulates cell migration and invasion via activating Stat3 in nasopharyngeal carcinoma
}

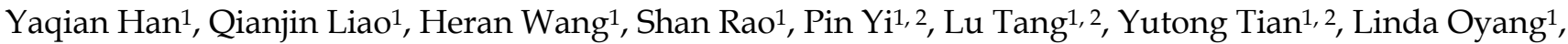 \\ Hui Wang ${ }^{1}$, Yingrui Shi ${ }^{\bowtie}$, Yujuan Zhou ${ }^{1 凶}$ \\ 1. Hunan Cancer Hospital and The Affiliated Cancer Hospital of Xiangya School of Medicine, Central South University, 283 Tongzipo Road, Changsha 410013, \\ Hunan, China. \\ 2. University of South China, Hengyang, 421001, Hunan, China \\ $\square$ Corresponding authors: Yujuan Zhou and Yingrui Shi, Hunan Cancer Hospital and The Affiliated Cancer Hospital of Xiangya School of Medicine, Central \\ South University, 283 Tongzipo Road, Changsha 410013, Hunan, China. Tel: 86-731-88651680; Fax: 86-731-88651999. Email: zhouyujuan@hnca.org.cn, \\ shiyingrui@ hnca.org.cn \\ (c) The author(s). This is an open access article distributed under the terms of the Creative Commons Attribution License (https://creativecommons.org/licenses/by/4.0/). \\ See http://ivyspring.com/terms for full terms and conditions.
}

Received: 2019.03.31; Accepted: 2019.08.12; Published: 2019.08.29

\begin{abstract}
Objective: Emerging evidence suggests that calreticulin (CALR) has great impacts on the tumor formation and progression of various cancers, but the role of CALR remains controversial. We investigated the expression and clinical significance of CALR in nasopharyngeal carcinoma (NPC).

Methods: Immunohistochemistry was used to detect the expression of CALR in NPC tissues, and the correlation of CALR with clinicopathological characteristics and prognosis were analyzed. The cell functions of CALR in NPC cells were also performed in vitro.

Results: Compared with non-tumor nasopharyngeal epithelium (NPE) tissues, CALR expression was markedly up-regulated in NPC tissues $(P<0.001)$, and the high expression of CALR was positively associated with advanced clinical stage $(P=0.003)$ and metastasis $(P=0.023)$. Compared to the patients with low expression of CALR, patients who displayed high expression of CALR may achieve a poorer progression-free survival (PFS) and overall survival $(O S)(P<0.001)$. Furthermore, multivariate analysis showed that high expression of CALR was an independent predictor of poor prognosis. In addition, we found that knockdown of CALR significantly inhibited the proliferation, migration and invasion of CNE2 and HONE1 cells in vitro, and the mechanism might be associated with inactivation of Stat3 signaling pathway.

Conclusion: CALR may promote NPC progression and metastasis via involving Stat 3 signaling pathway, and can be regarded as an effective potential predictor for progression and prognosis of NPC.
\end{abstract}

Key words: nasopharyngeal carcinoma; calreticulin; prognosis; progression; STAT3

\section{Introduction}

Nasopharyngeal carcinoma (NPC) is one type of head and neck malignancies, and has a notable high prevalence in southern China[1]. Multiple studies found that NPC is closely associated with EB virus infection, environment, genetic susceptibility ${ }^{[2-4]}$. Several potential biomarkers have been correlated with the outcome ${ }^{[4,5]}$. Nonetheless, these prognostic factors have limited utility in terms of predicting survival.

Calreticulin (CALR/CRT) protein is one of commonly expressed and highly conserved endoplasmic reticulum calcium protein[6], which can regulate multiple physiological function through regulating calcium balance, assisting proper protein 
folding, participating the processes of apoptosis, adhesion, angiogenesis and autoimmune reactions $\left[{ }^{[6,7]}\right.$. An increasing number of studies have demonstrated that CALR may be involved in the tumor initiation, progression, and metastasis, suggesting that CALR is a key therapeutic target in cancer ${ }^{[6-9]}$. CALR was highly expressed in gastric cancer, breast cancer, and other tumors, which was related to migration, invasion and abnormal apoptosis of tumor cells ${ }^{[10-12]}$. In gastric cancer, CALR can promote tumor angiogenesis, local invasion and lymph node metastasis, which is a good biomarker of prognosis in gastric cancer[13]. However, to date, the expression of CALR and associated clinical value are still unclear in NPC. In addition, our previous studies have indicated that CALR expression was down-regulated in response to LPLUNC1 (long palate, lung and nasal epithelium clone) overexpression, as detected using 2-D DIGE[14], and our studies have indicated that LPLUNC acts as a potential tumor suppressor gene in NPC [15-18]. Thus, there may be a close relationship between CALR and NPC, and we speculated that the CALR might play an important role in the occurrence and development of NPC. To verify the speculation, we investigated the expression of CALR in the NPC tissues and evaluated whether CALR might play a critical role in NPC progression and prognosis as a potential prognostic biomarker.

\section{Materials and Methods}

\section{Tumor samples}

As our previous study ${ }^{[14]}$, NPC samples (256 cases) and non-tumor nasopharyngeal epithelium (NPE) (131 cases) were collected in Xiangya Hospital and the Second Xiangya Hospital (Changsha, PR China) during 2002 to 2004. All biopsies were immediately fixed in $4 \%$ buffered paraformaldehyde, routinely processed and embedded in paraffin, and then prepared the NPC tissue microarray. The clinicopathologic characteristics were listed in Table 1. Among all of the NPC patients, we followed up 81 patients to do survival analysis. The time of following up was from 4 to 95 month, and average was 57 month.

For the mRNA expression study, another 36 NPC tissues and 15 NPE samples were obtained from patients in the Hunan Cancer Hospital/The Affiliated Cancer Hospital of Xiangya School of Medicine (Changsha, China) in 2013. All tissue samples were quickly frozen in liquid nitrogen and stored at $-80^{\circ} \mathrm{C}$ until laser-capture micro-dissection (LCM). We used a LEICA CM 1900 (Leica, Solms, Germany) for frozen sections and the Leica AS LMD system (Leica) to obtain the pure tissues.
All of the individuals participating in this project signed the informed consent form and their clinicopathologic characteristics, such as name, sex, age, metastasis and clinical stages were recorded.

\section{Cells and cell culture}

NP69 cell line and all the NPC cell lines (CNE1, CNE2, HONE1, C666-1, HNE1, HNE2 and 5-8F) were obtained from the Cancer Research Institute of Central South University. The cells were cultured in RPMI-1640 (Gibco, Logan, UT, USA). The media were supplemented with $12 \%$ FBS (Zeta Life, France), 100 $\mu \mathrm{g} / \mathrm{ml}$ penicillin, and $100 \mathrm{U} / \mathrm{ml}$ streptomycin (Gibco, Life Technologies, USA) at $37{ }^{\circ} \mathrm{C}$ in a $5 \% \mathrm{CO}_{2}$ incubator.

\section{Immunohistochemistry (IHC)}

Immunohistochemistry was done from paraffin-embedded tissue sections, and the CALR expression in individual NPC and NPE specimens was characterized by IHC using an immunohistochemistry kit (ComWin Biotech Co.,Ltd., Beijing, China) and following the manufacturer instructions. Slides were incubated with the primary antibody monoclonal rabbit anti- CALR (1:500, Abcam, Cambridge, UK) for overnight at $4^{\circ} \mathrm{C}$. Negative rabbit serum was used instead of the primary antibody as negative control. The specimens were visualized with 3, 3'-diaminobenzidine and counterstained with hematoxylin. The staining was brown or tan, and located in the cytoplasm or nucleus.

\section{Immunohistochemical evaluation}

A semiquantitative scoring criterion for $\mathrm{IHC}$ was used in which the staining intensity and positive areas were recorded as reported previously[14]. Briefly, the intensity of anti-CALR staining was scored by $0-3$, according to the standards of 0 (no staining), 1 (weak staining), 2 (medium staining), and 3 (strong staining). The percentage of reactivity was scored as follows: 0 (no positive tumor cells), 1 ( $<10 \%$ positive tumor cells), 2 (10-50\% positive tumor cells) and 3 ( $>50 \%$ positive tumor cells). Individual samples were evaluated by at least two pathologists in a blinded manner, and those samples with inconsistent scores were further discussed and decided. For the statistical analysis, the score was obtained by multiplying the intensity and reactivity rates. Scores of $<4$ suggested negative expression, and the remainder were classified as positive expression.

\section{Western blotting}

As reported previously[15], cells were lysed in RIPA buffer in the presence of Protease Inhibitor Cocktail and PhoSTOP (Roche, Basel, Switzerland). Protein was quantified using a BCA Protein Assay Kit 
(Pierce Biotechnology, Rockford, IL, USA). Protein $(30 \mu \mathrm{g})$ was separated using $10 \%$ sodium dodecyl sulfate polyacrylamide gel electrophoresis and transferred onto polyvinylidene fluoride membranes (PVDF) (Millipore, Billerica, MA, USA). The membranes were blocked with $5 \%$ non-fat milk in Tris-buffered saline and then incubated with primary antibodies at $4{ }^{\circ} \mathrm{C}$ overnight. Membranes were washed three times in TBST solution for $10 \mathrm{~min}$ each and then incubated with secondary antibodies. Signals were detected by an enhanced chemiluminescence detection system as the manufacturer's protocol (Bio-Rad, Hercules, CA, USA). The primary antibodies as follows: CALR (A1066\#) rabbit polyclonal antibody was purchased from ABclonal Biotechnology Co., Ltd (Hubei, China), Stat3 (12640\#) and p-Stat3 (9145\#) rabbit monoclonal antibody was purchased from Cell Signaling Technology (Boston, USA), GAPDH (60004-1-Ig) mouse monoclonal antibody was purchased from Proteintech Company (Chicago, USA).

\section{RNA isolation and real-time PCR}

Total RNA was isolated using Trizol (Invitrogen, CA) according to the manufacturer's instructions, and reversely transcribed into complementary DNA using AMV reverse transcriptase (Promega, San Luis Obispo, CA, USA). The levels of target gene mRNA transcripts were determined by qRT-PCR using specific primers and a SYBR-green-containing PCR kit (GenePharma, Shanghai, China). The sequences of primers were forward 5'-TCTCAGTTCCGGCAAG TTCT- $3^{\prime}$ and reverse $5^{\prime}$-TTCTGAGTCTCCGTGCA TGT-3' for CALR (232 bp); forward 5'-GAAGGTGAAGGTCGGAGTC- $3^{\prime}$ and reverse 5'-GAAGATGGTGAT GGGATTTC-3' for GAPDH (226 bp). The relative levels of individual gene mRNA transcripts to control GAPDH were determined.

\section{Cck-8 assay}

The Cck-8 assay was used to detect the proliferation rate of tumour cells. Cells of CNE2 and HONE1 were stably transfected with interfering plasmids and control vectors, respectively. Cells were trypsinized and seeded in 96-well plates at a density of $5 \times 10^{3}$ cells/well in $200 \mu \mathrm{l}$ of complete medium. Then, $10 \mu \mathrm{l}$ of $5 \mathrm{mg} / \mathrm{ml} \mathrm{CCK-8} \mathrm{(Sigma,} \mathrm{USA)} \mathrm{was}$ placed in each well at $0,24,48$, and $72 \mathrm{~h}$ and incubated at $37^{\circ} \mathrm{C}$ for $4 \mathrm{~h}$. The absorbance was measured at 460 nm.

\section{Cell migration and invasion assays}

A transwell chamber $(8 \mu \mathrm{m}, 24$-well format; Corning, USA), with or without a diluted Matrigel (BD Biosciences, New Jersey, USA) coating, was used to assess the migration and invasion of cultured cells.
Briefly, $5 \times 10^{4} / 200 \mu 1$ of cells (migration assays) and $1 \times 10^{5} / 200 \mu \mathrm{l}$ of cells (invasion assays) were seeded in serum-free RPMI-1640 to the top chamber, the cells in the top chamber that had not migrated through the filter were wiped off with a cotton swab, while those that had migrated to the bottom surface were fixed in $4 \%$ paraformaldehyde for $30 \mathrm{~min}$ and stained with $0.1 \%$ crystal violet, then counted under a microscope. Migration and invasion rates are expressed as the ratio of the treated group value to the control group value.

\section{Wound-healing assay}

Cells were seeded into 6-well plates (Corning, USA) in triplicate in adequate numbers for growth and attachment. The artificial "wound" was scratched by a $10 \mu l$ pipette tip after cells were grown to $80 \%$ confluence, washed gently in PBS until there were no floating cells and then incubated in medium containing 3\% FBS. Gap size was measured 0h, 24h, $48 \mathrm{~h}$, and the wound areas were then photographed using an inverted microscope.

\section{Statistical analysis}

All analyses were performed using SPSS 15.0 program for Windows software package (SPSS, Chicago, IL, USA). Statistical significance between groups within experiments was determined by the One-way ANOVA and Student t-test. The chi-square test was used to determine whether two groups had distinct gene expression levels. Survival was estimated using the Kaplan-Meier method and compared by log-rank test. Multivariate logistic analyses using a stepwise Cox regression model after adjusting for baseline characteristics. A P-value of < 0.05 was considered statistically significant.

\section{Results}

\section{CALR is overexpressed in NPC}

Immunohistochemistry was used to measure the expression of CALR in NPC and NPE tissues. CALR was positively expressed mainly in the cytoplasm, showing obvious pale brown. Representative photographs for immunostaining were shown in Fig. 1A. Only a total of $16.8 \%(22 / 131)$ was high expression in NPE tissues, while a total of $64.1 \%$ $(164 / 256)$ was high expression in NPC tissues $\left(X^{2}\right.$ $=77.564, \mathrm{P}<0.001$ ), suggesting CALR protein expression significantly increased in NPC tissues. Moreover, to further validate the level of CALR expression in the NPC tissues, we applied laser capture microdissection (LCM) to ensure the purity of NPC and NPE tissues, and detected the expression of CALR in mRNA level by qRT-PCR. Accord with the protein level, the mRNA expression of CALR was 
up-regulated significantly in NPC tissues $(\mathrm{P}<0.001$, Fig. 1B). Compared with normal human nasopharyngeal epithelium cell line (NP69), CALR was also overexpressed in four NPC cell lines (CNE1, CNE2, HONE1, C666-1, HNE1, HNE2 and 5-8F) (Fig. 1C). These results suggested that CALR was highly expressed in NPC, which related to the development and progression of NPC.

\section{Associations between CALR protein expression and clinicopathological parameters}

Next, to clarify the clinical significance of the expression of CALR in NPC, the relationship between the expression of CALR and clinicopathological parameters was analyzed. As shown in Table 1, the increased CALR expression appeared to be associated with advanced clinical stage $(\mathrm{P}=0.003)$ and metastasis $(\mathrm{P}=0.023)$ of NPC, while not significant with gender $(\mathrm{P}$ $=0.325)$ and age $(P=0.123)$. Therefore, these results suggested that high expression of CALR was related to the progression of NPC, CALR could represent predictive factors of NPC.

Table 1. Relationships between expression levels of CALR and clinicopathologic characteristics

\begin{tabular}{lllll}
\hline Characteristics & Cases & CALR & \\
\cline { 3 - 5 } & & High & Low & $\boldsymbol{P}$ Value \\
\hline Normal & 131 & 22 & 109 & \\
Age & & & & \\
$\leq 48$ & 66 & 10 & 56 & 0.612 \\
$>48$ & 65 & 12 & 53 & \\
Gender: & & & & \\
Male & 95 & 14 & 81 & 0.306 \\
Female & 36 & 8 & 28 & \\
Tumor & 256 & 164 & 92 & \\
Age & & & & \\
$\leq 48$ & 125 & 86 & 39 & 0.123 \\
$>48$ & 131 & 78 & 53 & \\
Gender: & & & & \\
Male & 200 & 125 & 75 & 0.325 \\
Female & 56 & 39 & 17 & \\
Metastasis & 164 & 116 & 48 & \\
No metastasis & 92 & 48 & 44 & 0.003 \\
Stages I+II & 115 & 65 & 50 & \\
Stages III+IV & 141 & 99 & 42 & 0.023 \\
\hline
\end{tabular}
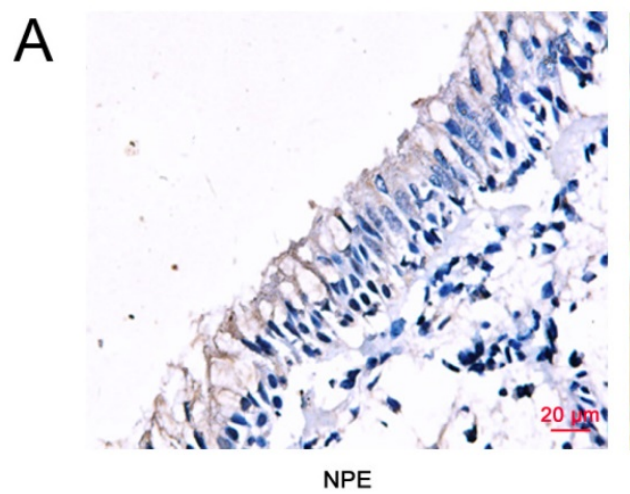

B
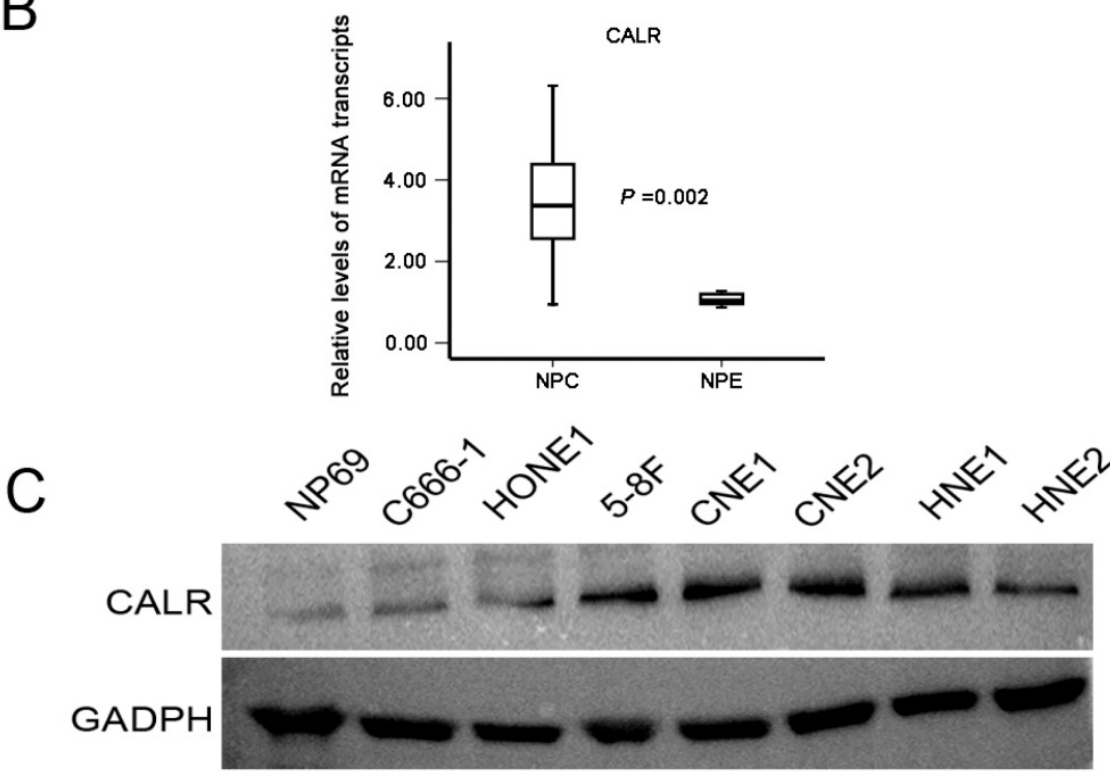

Figure 1. Expression of CALR in NPC tissues and cells. A: Representative IHC staining of CALR in NPC tissues (magnification $\times 400$, scale bars $20 \mu \mathrm{mm}$ ); $\mathrm{B}$ : qRT-PCR analysis of the relative expression levels of CALR in 36 NPC specimens and 15 NPE specimens; C: Detection the expression of CALR in NP69 cell line and NPC cell lines by western blotting. NPC: nasopharyngeal carcinoma, NPE: non-tumor nasopharyngeal epithelium. 


\section{CALR high expression correlated with poor prognosis of NPC}

To understand the relationship of prognosis with expression of CALR in NPC, Kaplan-Meier method analysis the survival curve. As shown in Fig. 2, the expression of CALR was closely associated with the progression-free survival (PFS) and overall survival (OS) of NPC. Patients with NPC having a low expression of CALR had a significantly longer PFS and OS compared with the patients having high expression $\left(61.023 \pm 3.735\right.$ vs $33.758 \pm 3.243, X^{2}=$ 14.901, $P<0.001 ; 77.111 \pm 4.697$ vs $39.785 \pm 3.884, X^{2}$ $=20.275, \quad P<0.001)$. Furthermore, multivariate regression analysis showed (Table 2) that CALR protein expression was significantly related to the PFS and OS, NPC patients who had high expression of CALR protein, had a shorter PFS $(\mathrm{HR}=2.564, \mathrm{P}=$ $0.013)$ and $\mathrm{OS}(\mathrm{HR}=3.179, \mathrm{P}=0.003)$, while the age, gender, metastasis and clinical staging had no significant association with PFS or OS of patients through COX multi-factor regression. These findings suggested that the high expression of CALR was a potential molecular marker for poor prognostic monitoring in NPC.

\section{Knockdown of CALR inhibited cell proliferation, migration and invasion in NPC cells}

We also explored the effect of CALR knockdown on NPC cell proliferation, migration and invasion. Cck8 assay showed that knockdown of CALR significantly inhibited the proliferation of CNE2 and HONE1 cells compared to the control group (Fig. 3A). The scratch wound-healing assay showed that compared with the empty vector group, knockdown of CALR in CNE2 and HONE1 cells led to slowly wound healing (Fig. 3B), and cell migration was significantly weakened (Fig. 3C). Matrigel invasion

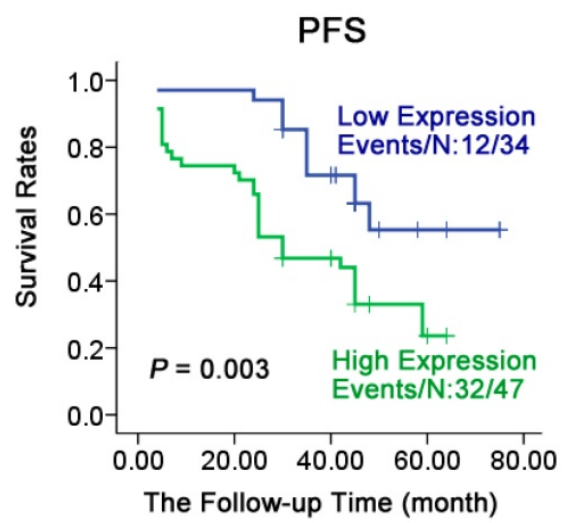

assays also showed that knockdown of CALR could significantly reduce the invasiveness of CNE2 and HONE1 cells (Fig. 3C). The results illustrated that the expression of CALR promoted CNE2 and HONE1 cell proliferation, migration and invasion.

Table 2. Cox regression analyses of the various factors associated with PFS and OS in NPC patients

\begin{tabular}{|c|c|c|c|c|c|c|}
\hline \multirow[t]{2}{*}{ Variables } & \multicolumn{3}{|c|}{ progression-free survival } & \multicolumn{3}{|c|}{ overall survival } \\
\hline & HR & $95 \% \mathrm{CI}$ & $\mathbf{P}$ & HR & $95 \% \mathrm{CI}$ & $\mathbf{P}$ \\
\hline Gender (Female/Male) & 0.546 & $0.248-1.200$ & 0.132 & 0.492 & $0.221-1.096$ & 0.083 \\
\hline Age $(\leq 48 />48)$ & 0.710 & $0.380-1.327$ & 0.283 & 0.595 & $0.316-1.120$ & 0.108 \\
\hline Stage(I+II/III+IV) & 2.430 & $0.810-7.289$ & 0.113 & 2.466 & $0.868-7.003$ & 0.090 \\
\hline $\begin{array}{l}\text { Metastasis } \\
\text { No metastasis }\end{array}$ & 0.529 & $0.169-1.655$ & 0.274 & 0.476 & $0.162-1.400$ & 0.178 \\
\hline CALR(High/Low) & 2.564 & $1.217-5.405$ & 0.013 & 3.179 & $1.464-6.902$ & 0.003 \\
\hline
\end{tabular}

HR, hazard ratio; $95 \%$ CI, 95\% confidence interval

\section{Knockdown of CALR suppressed Stat3 signaling pathways in CNE2 and HONE1 cells}

Given to CALR is an upstream regulator of signal transducer and activator of transcription 3 (Stat3) ${ }^{[19]}$, we detected whether knockdown of CALR suppressed the activation of Stat3 signaling pathways in CNE2 and HONE1 cells. As illustrated in Fig. 4, the expression of Stat3 was detected by western blotting. Compared with the empty vector group, the levels of total Stat3, p-Stat3 protein expression significantly decreased in the CALR silencing CNE2 and HONE1 cells. The result indicated that CALR expression could activate Stat 3 signaling pathway, thereby promoting the cell proliferation, migration and invasion of CNE2 and HONE1cells.

\section{Discussion}

NPC is one kind of head and neck cancer that arise from cells in nasopharynx, which is typically diagnosed at advanced clinical stages, and resulting in poor outcomes ${ }^{[1]}$. Thus, it is important to explore the potential biomarkers for the diagnosis, prognosis, and

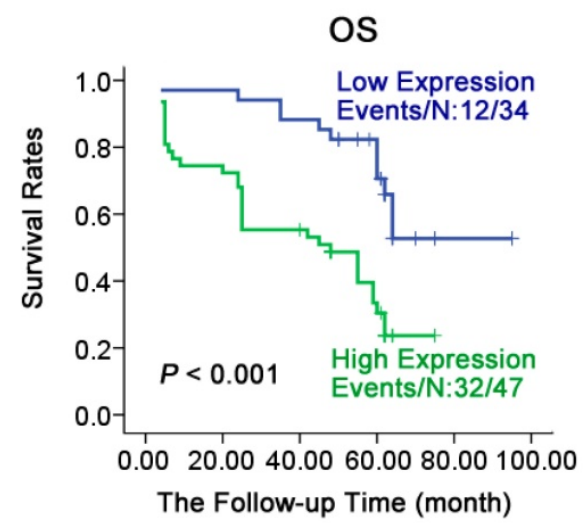

Figure 2. Survival analysis of patients with varying levels of CALR expression in NPC. Kaplan-Meier estimated progression-free survival (PFS) and overall survival (OS) for NPC patients according to the expression levels of CALR protein in 81 NPC patients. P values were obtained by using the log-rank test. N, The number of cases; Events, the number of cases, who had recurrent tumor or died during the follow-up period. 
treatment of NPC. In this study, CALR has been preliminarily verified as a potential molecular marker in NPC.

Our studies have indicated that LPLUNC could work as a potential tumor suppressor gene in NPC [15, $16,18]$, and we identified that CALR was one of differentially expressed genes using proteomic techniques in LPLUNC1 overexpression NPC cells, which was down-regulated by LPLUNC1, suggesting CALR plays an important role in the development of NPC and might be involved in the inhibitory roles of LPLUNC1 in NPC[14]. CALR (CRT) protein is one of the major calcium-binding protein in endoplasmic reticulum. CALR can locate in the endoplasmic reticulum, also locate in membrane and secret out of the cell. The biological function is mainly involved in the regulation of calcium balance, properly folded proteins, apoptosis, adhesion, and physiological and pathological processes of angiogenesis and autoimmune reactions ${ }^{[7,20]}$. Studies have shown that CALR protein expresses in a variety of tumor cell surface, which considered as an "eat-me" signal and promotes phagocytic uptake of cancer cells by immune system, resulting in inducing immunogenic
A
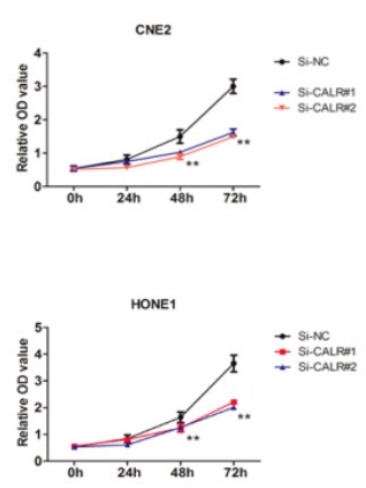

C

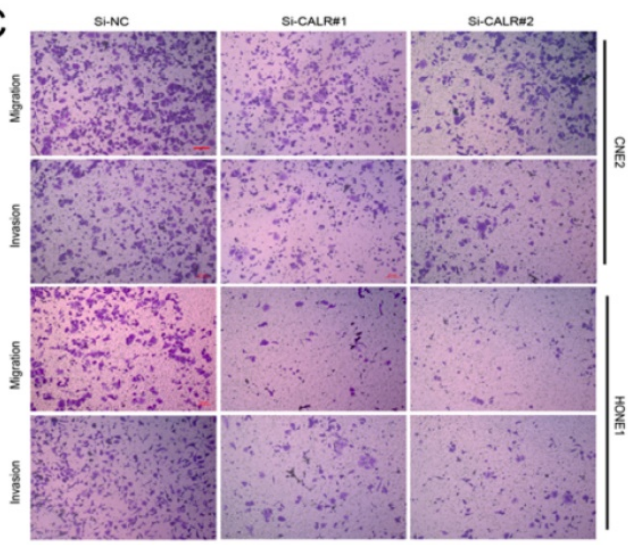

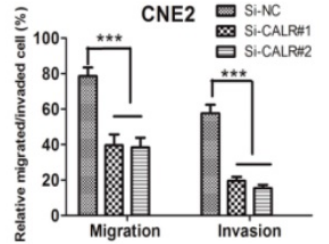

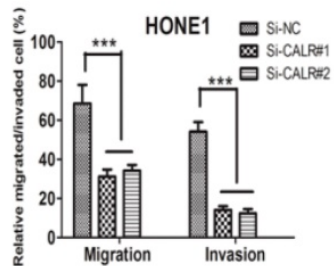

B

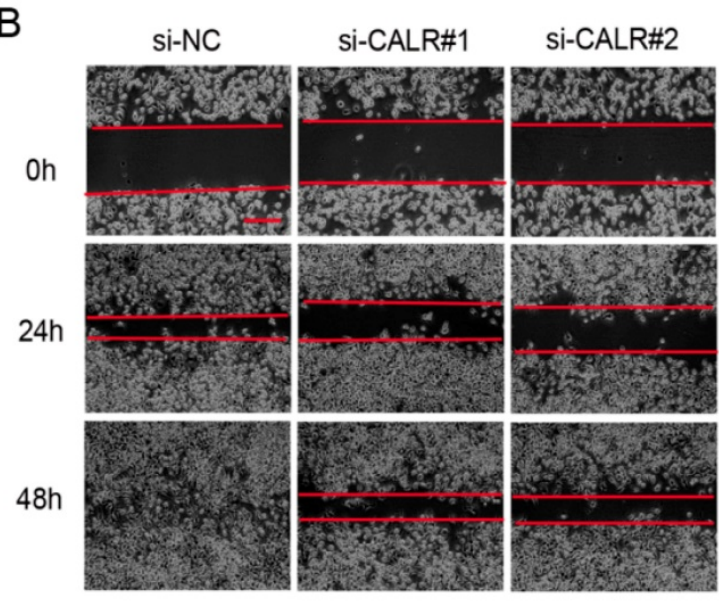

CNE2

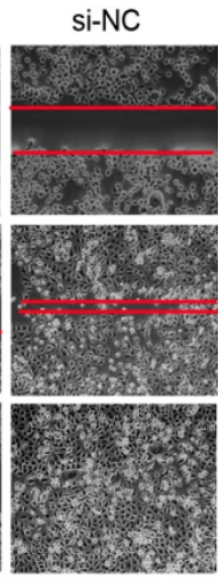

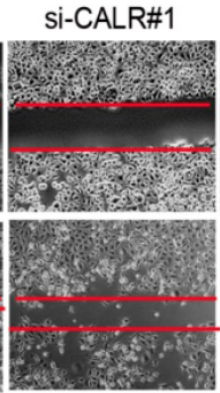

Si-CALR\#2

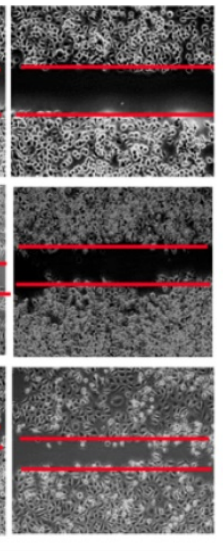

HONE1
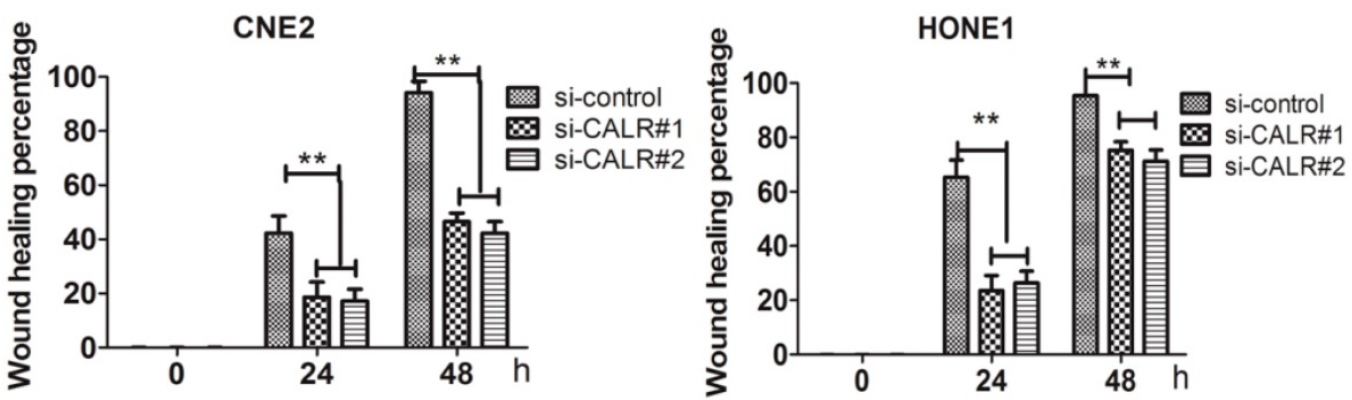

Figure 3. Knockdown of CALR inhibits the cell proliferation, migration and invasion of NPC cells. In order to silence the expression of CALR, CNE2 and HONE1 cells were transfected with CALR interfering plasmids (si-CALR\#1, si-CALR\#2). The proliferation of CNE2 and HONE1 cells was analyzed by Cck-8 assay (A), cell migration of CNE2 and HONE1 cells was analyzed using scratch wound assays (B), and further confirmed by transwell assays without a diluted Matrigel coating (C). Cell invasion of CNE2 and HONE1 cells was analyzed using transwell assays with a diluted Matrigel coating $(\mathrm{C})$. Error Bar $=\mathrm{SD}, * * \mathrm{P}<0.01$, ***P $<0.001$ compared to vector, independent Student's t-test. 
apoptosis and triggers anti-tumor immune responses $[8,10,21-23]$. Recently, increasing evidences also indicate that CALR has great impacts on the tumor formation and progression of various cancers, manipulation of CALR levels profoundly affects cancer cell proliferation and angiogenesis $[10,21,22]$, but the role of CALR in cancer cells remains controversial[21]. Many reports reveal that CALR protein is highly expressed in esophageal cancer[24], pancreatic cancer[25, 26], breast cancer[27], bladder cancer ${ }^{[12,28]}$ and other tumors, suggesting CALR plays a protumorigenic role. While downregulation of CALR has also been observed in endometrial cancer ${ }^{[29],}$ colon adenocarcinomas ${ }^{[30],}$ prostate cancer[31], showing CALR has anti-tumourigenic activity. Thus, the relationship between CALR and cancer requires further investigation. Considering the current evidence, the role of CALR expression in different tumors is unclear, especially, it remains unclear whether CALR may act as a biomarker for NPC patients or not. In this study, we examined the relationship between alterations in CALR expression and the prognosis of patients with NPC. We found that the expression of CALR was significantly increased in NPC tissues, suggesting that CALR expression plays a protumorigenic role in NPC.

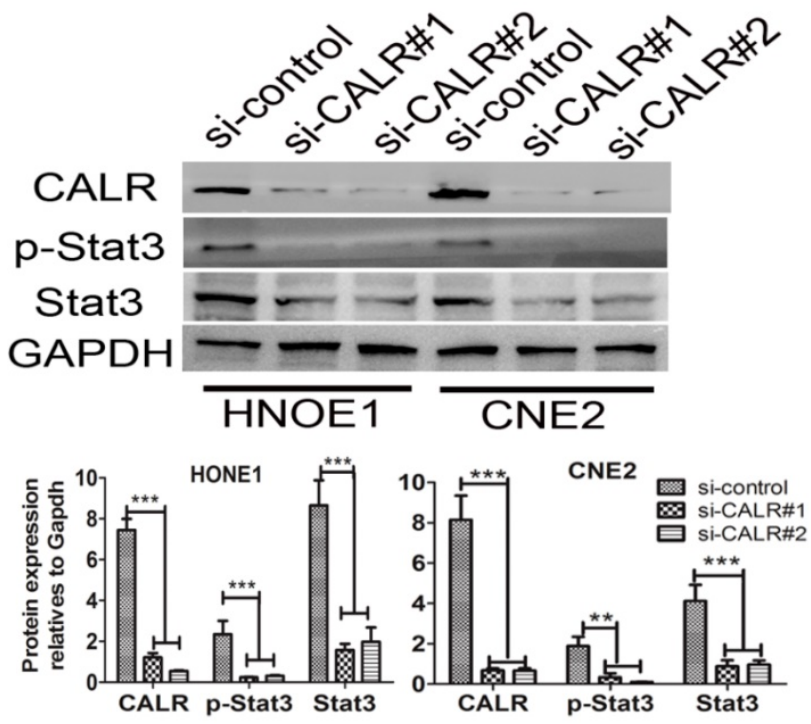

Figure 4. Knockdown of CALR suppressed the expression of Stat3 in NPC cells. The expression of CALR, Stat3 and p-Stat3 in CNE2 and HONE1 cells transfected with CALR interfering plasmids (si-CALR\#I, si-CALR\#2) and control vector were detected by western blotting.

The expression level of CALR was apparently related with the pathological grade of the tumor, lymph node metastasis and high microvessel density in various cancer, and resulted in poor patient survival [23,32]. It means that CALR may promote tumor angiogenesis, accelerate tumor invasion and metastasis ${ }^{[24]}$ CALR overexpression enhanced angiogenesis, migration and metastasis of gastric cancer cells ${ }^{[13,33]}$. High expression of CALR is highly associated with invasion and metastasis, apoptosis resistance and poor prognosis in esophageal squamous cell carcinoma (ESCC), the capabilities of ESCC movement, invasion and resistant to apoptosis was significantly decreased after blocking endogenic expression of CALR [24, 34]. CALR expression was also higher in the more aggressive MDA-MB-231 cells compared with MCF-7 cells at both the mRNA and protein levels[35, 36], siRNA-mediated knockdown of CRT expression significantly decreased invasiveness of DADS-treated HL-60 cells[37, 38]. In this study, we analyzed the clinical significance of CALR expression in NPC tissues. Consistent with these reports, we found that the expression of CALR was positively correlated with the clinical stage and metastasis in NPC. Survival analysis showed over-expressed CALR in NPC patients had poor prognosis, with a shorter PFS and OS. COX multivariate regression analysis showed high expressions of CALR is important biomarkers associated with poor prognosis in NPC patients. Furthermore, we found that knockdown of CALR significantly inhibited the proliferation, migration and invasion of CNE2 and HONE1 cells. The results suggested that CALR played important roles in morbidity and progression of NPC.

Numerous studies have demonstrated that CALR is an upstream regulator of Stat3[11, 19]. In esophageal squamous cell carcinoma, CALR works as the upstream regulator of STAT3 promotes cell motility and enhances resistance to anoikis ${ }^{[34]}$. It is well known that Stat3 is a point of convergence for numerous oncogenic signaling pathways ${ }^{[39-41]}$. Stat 3 is constitutively activate in both tumor cells and immune cells, and this activation promotes tumor survival and growth by increasing the capacity of tumors to evade the immune system[40, 42, 43]. Interestingly, our previous study has demonstrated that LPLUNC1 overexpression significantly inhibited Stat3 signaling pathway induced by IL-6[18], while CALR was down-regulated by LPLUNC1 ${ }^{114]}$. Therefore, there may be a close relationship between CALR and Stat3 in NPC, but the regulatory effect of CALR on Stat 3 is still unclear. In the current study, we found that knockdown of CALR could significantly suppress the activation of Stat 3 signaling pathway in CNE2 and HONE1 cells, suggesting CALR could activate Stat3, CALR-Stat3 signaling pathway has been involved in the initiation and progress of NPC and the inhibitory roles of LPLUNC1 in NPC, which will be further investigated in the future.

In summary, we found that CALR was significantly up-regulated in NPC tissues, CALR high expression was positively related to advanced clinical 
stage, metastasis and poorer prognosis in NPC. Knockdown of CALR significantly inhibited the proliferation, migration and invasion via inactivation of Stat3 signaling pathway in CNE2 and HONE1 cells. CALR may be regarded as biomarkers of malignant progression and poor prognosis, which might become novel therapeutic targets to prevent the progression of NPC. However, the function of CALR in NPC need to be further investigated in vivo and in vitro.

\section{Abbreviations}

CALR/CRT: calreticulin; NPC: nasopharyngeal carcinoma; PFS: progression-free survival; OS: overall survival; LPLUNC1: long palate, lung, and nasal epithelium clone; NPE: non-tumor nasopharyngeal epithelium; IHC: Immunohistochemistry; Stat3: signal transducer and activator of transcription 3.

\section{Acknowledgements}

This work was supported in part by grants from the following sources: the National Natural Science Foundation of China $(81472595,81872281$, the Natural Science Foundation of Hunan Province (2019JJ40175, 2018JJ1013, 2017JJ3190, 2016JJ4059), the Research Project of the Health and Family Planning Commission of Hunan Province (B20180400, B20180582), and the Changsha Science and Technology Board (kq1706045, kq1706043), Beijing Hope Run Special Fund of Cancer Foundation of China (LC2016W06), Ascend Foundation of National cancer center (NCC2018b68).

\section{Ethics Committee Approval and Patient Consent}

This study was approved by the ethics committee of Hunan Cancer Hospital/The Affiliated Cancer Hospital of Xiangya School of Medicine. All individuals participating in this project signed the informed consent.

\section{Competing Interests}

The authors have declared that no competing interest exists.

\section{References}

[1] Xu C, Chen YP, Ma J. Clinical trials in nasopharyngeal carcinoma-past, present and future. Chin Clin Oncol, 2016,5(2):20.

[2] Fan C, Tang Y, Wang J, et al. The emerging role of Epstein-Barr virus encoded microRNAs in nasopharyngeal carcinoma. J Cancer, 2018,9(16):2852-2864.

[3] Huang SCM, Tsao SW, Tsang CM. Interplay of Viral Infection, Host Cell Factors and Tumor Microenvironment in the Pathogenesis of Nasopharyngeal Carcinoma. Cancers (Basel), 2018,10: 4.

[4] Zhou Y, Xia L, Lin J, et al. Exosomes in Nasopharyngeal Carcinoma. J Cancer, 2018,9(5):767-777.

[5] Paul P, Deka H, Malakar AK, et al. Nasopharyngeal carcinoma: understanding its molecular biology at a fine scale. Eur J Cancer Prev, 2018,27(1):33-41.

[6] Eggleton P, Bremer E, Dudek E, et al. Calreticulin, a therapeutic target? Expert Opin Ther Targets, 2016,20(9):1137-47.

[7] Jiang Y, Dey S, Matsunami H. Calreticulin: roles in cell-surface protein expression. Membranes (Basel), 2014,4(3):630-41.
[8] Holmstrom MO, Martinenaite E, Ahmad SM, et al. The calreticulin (CALR) exon 9 mutations are promising targets for cancer immune therapy. Leukemia, 2018,32(2):429-437.

[9] Korbelik M, Banath J, Saw KM, et al. Calreticulin as cancer treatment adjuvant: combination with photodynamic therapy and photodynamic therapy-generated vaccines. Front Oncol, 2015,5: 15.

[10] Sun J, Mu H, Dai K, et al. Calreticulin: a potential anti-cancer therapeutic target. Pharmazie, 2017,72(9):503-510.

[11] Zamanian M, Veerakumarasivam A, Abdullah S, et al. Calreticulin and cancer. Pathol Oncol Res, 2013,19(2):149-54.

[12] Lu YC, Chen CN, Wang B, et al. Changes in tumor growth and metastatic capacities of J82 human bladder cancer cells suppressed by down-regulation of calreticulin expression. Am J Pathol, 2011,179(3):1425-33.

[13] Chen CN, Chang CC, Su TE, et al. Identification of calreticulin as a prognosis marker and angiogenic regulator in human gastric cancer. Ann Surg Oncol, 2009,16(2):524-33.

[14] Zhou Y, Liao Q, Li X, et al. HYOU1, Regulated by LPLUNC1, Is Up-Regulated in Nasopharyngeal Carcinoma and Associated with Poor Prognosis. J Cancer, 2016,7(4):367-76

[15] Wang H, Zhou Y, Oyang L, et al. LPLUNC1 stabilises PHB1 by counteracting TRIM21-mediated ubiquitination to inhibit NF-kappaB activity in nasopharyngeal carcinoma. Oncogene, 2019, 38(25):5062-5075.

[16] Wei F, Tang L, He Y, et al. BPIFB1 (LPLUNC1) inhibits radioresistance in nasopharyngeal carcinoma by inhibiting VTN expression. Cell Death Dis, 2018,9(4):432.

[17] Wei F, Wu Y, Tang L, et al. BPIFB1 (LPLUNC1) inhibits migration and invasion of nasopharyngeal carcinoma by interacting with VTN and VIM. Br J Cancer, 2018,118(2):233-247.

[18] Liao Q, Zeng Z, Guo X, et al. LPLUNC1 suppresses IL-6-induced nasopharyngeal carcinoma cell proliferation via inhibiting the Stat3 activation. Oncogene, 2014,33(16):2098-109.

[19] Jiao Y, Ding H, Huang S, et al. Bcl-XL and Mcl-1 upregulation by calreticulin promotes apoptosis resistance of fibroblast-like synoviocytes via activation of PI3K/Akt and STAT3 pathways in rheumatoid arthritis. Clin Exp Rheumatol, 2018,36(5):841-849.

[20] Feng M, Marjon KD, Zhu F, et al. Programmed cell removal by calreticulin in tissue homeostasis and cancer. Nat Commun, 2018,9(1):3194.

[21] Venkateswaran K, Verma A, Bhatt AN, et al. Emerging Roles of Calreticulin in Cancer: Implications for Therapy. Curr Protein Pept Sci, 2018,19(4):344-357.

[22] Lu YC, Weng WC and Lee H. Functional roles of calreticulin in cancer biology. Biomed Res Int, 2015,2015: 526524.

[23] Fucikova J, Kasikova L, Truxova I, et al. Relevance of the chaperone-like protein calreticulin for the biological behavior and clinical outcome of cancer. Immunol Lett, 2018,193: 25-34.

[24] Shi F, Shang L, Pan BQ, et al. Calreticulin promotes migration and invasion of esophageal cancer cells by upregulating neuropilin-1 expression via STAT5A. Clin Cancer Res, 2014,20(23):6153-62.

[25] Matsukuma S, Yoshimura K, Ueno T, et al. Calreticulin is highly expressed in pancreatic cancer stem-like cells. Cancer Sci, 2016,107(11):1599-1609.

[26] Sheng W, Chen C, Dong M, et al. Overexpression of calreticulin contributes to the development and progression of pancreatic cancer. J Cell Physiol, 2014,229(7):887-97.

[27] Zamanian M, Qader Hamadneh LA, Veerakumarasivam A, et al. Calreticulin mediates an invasive breast cancer phenotype through the transcriptional dysregulation of p53 and MAPK pathways. Cancer Cell Int, 2016,16: 56.

[28] Iwaki H, Kageyama S, Isono T, et al. Diagnostic potential in bladder cancer of a panel of tumor markers (calreticulin, gamma -synuclein, and catechol-o-methyltransferase) identified by proteomic analysis. Cancer Sci, 2004,95(12):955-61.

[29] Xu Q, Chen C, Chen G, et al. Significance of calreticulin as a prognostic factor in endometrial cancer. Oncol Lett, 2018,15(6):8999-9008.

[30] Toquet C, Jarry A, Bou-Hanna C, et al. Altered Calreticulin expression in human colon cancer: maintenance of Calreticulin expression is associated with mucinous differentiation. Oncol Rep, 2007,17(5):1101-7.

[31] Alur M, Nguyen MM, Eggener SE, et al. Suppressive roles of calreticulin in prostate cancer growth and metastasis. Am J Pathol, 2009,175(2):882-90.

[32] Liu R, Gong J, Chen J, et al. Calreticulin as a potential diagnostic biomarker for lung cancer. Cancer Immunol Immunother, 2012,61(6):855-64.

[33] Liu SH, Lee WJ, Lai DW, et al. Honokiol confers immunogenicity by dictating calreticulin exposure, activating ER stress and inhibiting epithelial-to-mesenchymal transition. Mol Oncol, 2015,9(4):834-49.

[34] Du XL, Yang H, Liu SG, et al. Calreticulin promotes cell motility and enhances resistance to anoikis through STAT3-CTTN-Akt pathway in esophageal squamous cell carcinoma. Oncogene, 2009,28(42):3714-22.

[35] Lwin ZM, Guo C, Salim A, et al. Clinicopathological significance of calreticulin in breast invasive ductal carcinoma. Mod Pathol, 2010,23(12):1559-66.

[36] Chen Q, Fang X, Jiang C, et al. Thrombospondin promoted anti-tumor of adenovirus-mediated calreticulin in breast cancer: Relationship with anti-CD47. Biomed Pharmacother, 2015,73: 109-15.

[37] Sun J, Mu H, Yu J, et al. Diallyl disulfide down-regulates calreticulin and promotes C/EBPalpha expression in differentiation of human leukaemia cells. J Cell Mol Med, 2019,23(1):194-204.

[38] Yi L, Shan J, Chen X, et al. Involvement of calreticulin in cell proliferation, invasion and differentiation in diallyl disulfide-treated HL-60 cells. Oncol Lett, 2016,12(3):1861-1867 
[39] Huynh J, Chand A, Gough D, et al. Therapeutically exploiting STAT3 activity in cancer - using tissue repair as a road map. Nat Rev Cancer, 2019,19(2):82-96.

[40] Guanizo AC, Fernando CD, Garama DJ, et al. STAT3: a multifaceted oncoprotein. Growth Factors, 2018,36(1-2):1-14.

[41] Fathi N, Rashidi G, Khodadadi A, et al. STAT3 and apoptosis challenges in cancer. Int J Biol Macromol, 2018,117: 993-1001.

[42] Roca Suarez AA, Van Renne N, Baumert TF, et al. Viral manipulation of STAT3: Evade, exploit, and injure. PLoS Pathog, 2018,14(3):e1006839.

[43] Johnson DE, O'keefe RA and Grandis JR. Targeting the IL-6/JAK/STAT3 signalling axis in cancer. Nat Rev Clin Oncol, 2018,15(4):234-248. 